\title{
Principal Oscillation Pattern Analysis of the 30- to 60-Day Oscillation in General Circulation Model Equatorial Troposphere
}

\author{
Hans von Storch, Thomas Bruns, Irene Fischer-Bruns, and Klaus Hasselmann \\ Max-Planck-Institut für Meteorologie, Hamburg, Federal Republic of Germany
}

\begin{abstract}
A new technique is described for identifying time-dependent patterns (i.e., "principal oscillation patterns," or POPs) in a set of geophysical time series. POPs are defined as the normal modes of a linear dynamical representation of the data in terms of a first-order autoregressive vector process with residual noise forcing. POPs associated with real eigenvalues represent nonpropagating, nonoscillatory patterns which decay exponentially. POPs associated with complex eigenvalues occur in complex conjugate pairs and can represent standing wave structures (if one pattern is much stronger than the other), propagating waves (if both patterns are periodic and have the same structure except for a quarter-wavelength shift) or, in general, an arbitrary amphidromal oscillation. After the POPs have been defined for a selected set of primary variables, associated correlation or composite patterns may be derived for additional secondary fields to gain further insight into the structure of the interaction mechanisms. The method is illustrated by analyzing the tropical variability structure of a 10-year numerical simulation with the T21 general circulation model (GCM) of the European Centre for Medium Range Weather Forecasts, Reading, England. The POP analysis is applied to the 200-hPa horizontal divergence field along the equator for time scales shorter than 10 weeks. The associated patterns are determined for a number of additional fields in the tropical belt between $30^{\circ} \mathrm{S}$ and $30^{\circ} \mathrm{N}$. One dominant POP pair is found. Its spatial scale corresponds to zonal wave number 1 . The patterns travel eastward, with an average period of 24 days and an $e$-folding decay time of 10 days. The maximum variance occurs in the area with maximum tropical precipitation, between $100^{\circ} \mathrm{E}$ and the dateline. These features correspond rather closely to the characteristics of the observed tropical "30- to 60-day wave," except for the smaller period. A frequencywave number analysis confirms that this 30- to 60-day wave is the most dominant regular oscillation in the tropical GCM troposphere. The associated patterns of sea level pressure, precipitation, and other quantities exhibit a number of intriguing aspects, namely, (1) the phase velocity of the 30- to 60-day wave varies with longitude from $6 \mathrm{~m} \mathrm{~s}^{-1}$ in the Indonesian area to more than $30 \mathrm{~m} \mathrm{~s}^{-1}$ over the eastern Pacific, small phase speeds being associated with large amplitudes and high phase speeds with small amplitudes; (2) in the high-amplıtude regions, rainfall and upper air velocity potential are in phase, while in the low-amplitude regions, rainfall and velocity potential appear uncorrelated; (3) at the surface a pattern strongly resembling Gill's theoretical response to an equatorial heating source is found, with a trough to the east and two off-equatorial cyclones to the west of the heating. The lack of meridional winds is probably related to the disregard of the seasonal asymmetries.
\end{abstract}

\section{INTRODUCTION}

\subsection{Principal Oscillation Pattern Analysis}

In this paper we describe and apply a new technique for analyzing spatial and temporal variability in a multicomponent data set. The "principal oscillation pattern" (POP) analysis identifies coherent migrating, standing, or otherwise changing patterns of the system without prior knowledge of the system dynamics. The basic approach is to represent the vector time series as the output of a multivariate first-order autoregressive process forced by residual noise (not necessarily white noise). The process matrix is estimated from the data. The POPs are defined as the real or complex eigenvectors of the process matrix, with associated real or complex eigenvalues. Complex POPs occur in complex conjugate pairs and represent damped amphidromal oscillations (including propagating and standing waves as special cases), while real POPs occur as singlets and describe nonpropagating, nonoscillating, damped patterns. In the continuous case the POP analysis corresponds to the normal-mode analysis of a linear system of differential equations.

Copyright 1988 by the American Geophysical Union.

Paper number 8D0305.

0148-0227/88/008D-0305\$05.00
The method described here represents the linearized form of the more general "principal interaction pattern" (PIP) technique for constructing simplified dynamical models to approximate complex nonlinear systems with many degrees of freedom [cf. Hasselmann, this issue]. In the present application, however, we emphasize the diagnostic rather than the dynamical aspects of the method. Thus we introduce a further simplification of the general technique in addition to the linearization. Rather than simultaneously seeking both the optimal linear model (i.e., the Markov system matrix) and the optimal vector subspace in which the model is to be constructed, in accordance with the general PIPs and POPs philosophy, we regard the vector subspace as given. We carry out a standard reduction of the number of variables by using a relatively small set of empirical orthogonal functions (EOFs) which captures most of the variance. The POP analysis is then carried out in this prescribed vector subspace.

The present analysis may be formally related to the canonical correlation analysis of Barnett and Preisendorfer [1987] and can be compared with a complete complex spectral EOF analysis [cf. Hasselmann, this issue]. It also has some analogy with the complex EOF (Hilbert transform) analysis applied by Barnett [1983]. Both the Hilbert transform and the present technique yield information not only on the variance, but also on the time dependence of the fields. But, in both cases, only partial information on the time dependence is provided, in the form of integral moments of the covariance cross spectra. 
Whereas the Hilbert transform uses straightforward unweighted spectral integrals, however, the POP technique extracts additional information about the peaks and peak widths of the cross spectra and thus yields not only the pattern structures, but also their characteristic frequencies and damping factors.

After the dominant POPs have been identified for selected field variables, "associated patterns" of the POPs may be derived for additional field variables, using either correlation or composite techniques, to gain additional insight into the general structure of the interaction processes. This two-step approach is essentially equivalent to (but computationally somewhat simpler than) carrying out a one-step POP analysis for all field variables simultaneously, with a large weighting being assigned to the selected principal fields and a weak weighting being assigned to the associated fields.

The general POP and associate pattern analysis techniques are described in more detail in section 2.

\subsection{Application to the Equatorial 30- to 60-Day Wave}

The POP method is illustrated by an analysis of the intraseasonal fluctuations of the equatorial troposphere for a 10year climate simulation carried out with the European Centre for Medium Range Weather Forecasts (ECMWF) T21 general circulation model (GCM).

Observations indicate that the intraseasonal variability of the zonal wind, sea level pressure, and outgoing longwave radiation in the tropics is dominated by global-scale seasonally modulated oscillations in the 30- to 60-day band [Madden and Julian, 1971, 1972; Madden, 1986; Lau and Chan, 1985; Knutson and Weickmann, 1987]. Since the oscillations originate predominantly in the Indonesian heating region, usually propagate eastward with a zonal wave number 1 structure, and have their largest amplitudes near the equator, they are believed to represent Kelvin waves induced by strong outbreaks of convective activity [Chang, 1977]. The Kelvin modes appear to be characterized by significantly longer life times than the westward traveling Rossby modes, which are presumably also generated together with the Kelvin mode but have not in fact been observed. Lau and Peng [1986] suggest that the conditional instability of the second kind (CISK) mechanism, in which low-level convergence provides enough humidity to sustain moist convection, could enable the Kelvin waves to propagate completely around the globe. Similar waves, although with shorter time scales (25-40 days), have also been found in GCM simulations [Lau and Lau, 1986; Lau, 1986; Geisler and Pitcher, 1986; Hayashi and Golder, 1986; Swinbank, 1986; Chiba, 1986]. Their occurrence in GCM simulations provides an opportunity to study the properties of the waves and their role in intra-annual variability under relatively controlled conditions [Lau and Chan, 1985].

We decided to analyze this phenomenon in the ECMWF T21 simulation for two reasons:

1. It provides an interesting test of the POP analysis method. Since the oscillation is a fairly well-defined, zonally distributed feature, it can also be investigated using standard frequency-wave number analysis methods [Hayashi, 1982]. Thus the results of the POP analysis may be validated by an established alternative, conceptually different statistical technique.

2. The 30- to 60-day wave represents an important physical phenomenon in its own right. It interacts with the monsoons and the trade wind system, induces low-frequency variability in the extratropics (on time scales of weeks) through teleconnections, and may be one of the quasi-random processes of the equatorial troposphere triggering the development of El Niño/Southern Oscillation (ENSO) events. Thus the ability of a GCM to simulate this wave is an important model test which should be carried out for any model used for studies of the tropical circulation, the ENSO phenomenon, or extratropical variability.

\section{Principal Oscillation Patterns}

POPs represent the linear, time-independent case of principal interaction patterns, which may be introduced generally to approximate nonlinear, time-dependent systems with many degrees of freedom [Hasselmann, this issue]. In the general approach a complex, multicomponent system is approximated by a hierarchy of simpler models containing only a small number of degrees of freedom and a corresponding number of interaction patterns. The number of degrees of freedom is successively increased in the hierarchy sequence. At each level of the hierarchy the dynamical model and the associated principal interaction patterns defining the system subspace in which the model operates are optimized simultaneously.

In the present context, however, we shall not be concerned with a model hierarchy, but with a single model only. This is presumed to be defined for a reasonably complete previously prescribed system subspace. For convenience we chose as our base vector space the standard truncated EOF space. In this case we may define POPs simply as the eigenmodes of the linear system obtained by fitting a first-order multivariate Markov process to the data in the truncated EOF space.

\subsection{Normal Modes}

Let $x(t)$ represent the $n$-dimensional stochastic vector process after reduction of the dimension of the original process by projection on to the truncated EOF space. The evolution of the process $x$ can always be represented as a linear process $d x / d t=A x+r$ with a constant matrix $A$ driven by (suitably defined, in general, nonwhite, non-Gaussian) noise $r(t)$. We assume that the forcing has zero mean, $\langle r\rangle=0$. Discretizing the time derivative, the process may be written as

$$
x(t+1)=B x(t)+r(t)
$$

with a constant matrix $B$ depending on the details of the time discretization scheme (examples are given by von Storch [1980]).

In most cases the homogenous system (1), with $r=0$, will have a complete set of linearly independent eigenmodes, with eigenvectors $p^{k}, k=1, \cdots, n$ and associated eigenvalues $\lambda^{k}$, $k=1, \cdots, n$ (degenerate cases will not be considered):

$$
B p^{k}=\lambda^{k} p^{k}
$$

Normally, $B$ is not symmetric, so that some, or possibly all, eigenvalues are complex. Since $x$ and $B$ are real, complex eigenvectors and eigenvalues occur in complex conjugate pairs.

The vector $x(t)$ can be represented in terms of the eigenvec- 
tors,

$$
x(t)=\sum_{k=1}^{n} a^{k}(t) p^{k}
$$

where the normal-mode coordinates $a^{k}(t)$ satisfy the standard diagonalized damped harmonic oscillator equations. In discrete notation,

$$
a^{k}(t+1)=\lambda^{k} a^{k}(t)+r^{k}
$$

where $r^{k}$ represents the forcing.

The solutions of the homogenous equations are given by

$$
a^{k}(t)=\text { const } \exp \left(-t / \tau_{k}\right) \exp \left(i 2 \pi t / T_{k}\right)
$$

with

$$
\begin{gathered}
\tau_{k}=-1 / \ln \left|\lambda^{k}\right| \\
T_{k}=2 \pi / \tan ^{-1}\left(\omega^{k} / \mu^{k}\right)
\end{gathered}
$$

and

$$
\lambda^{k}=\mu^{k}+i \omega^{k}
$$

\subsection{Representation of the Normal Modes}

The complex eigenvectors $p^{k}$ and normal-mode coordinates $a^{k}$ are defined only to within arbitrary complex normalization factors $m^{k} \exp \left(i \Theta^{k}\right)$. In the following discussion we consider one complex eigenvector $v^{k}$ and omit the index $k$.

The modulus $m$ of the normalization factors can be specified by requiring, for example, that

$$
\left(v^{*}, v\right)=1
$$

where ( , ) denotes some suitably defined scalar product in the space of state vectors and $v^{*}$ denotes the complex conjugate. (Note that we cannot generalize (6) to require orthogonality for different POPs, since the POPs are in general nonorthogonal for a nonsymmetrical system matrix $B$ ).

This still leaves the phase $\Theta$ of the normalization factor free. A natural choice of phase angle in the complex plane is to require that the real and imaginary components of the complex pattern $p, p_{1}$ and $p_{2}$, respectively, are orthogonal,

$$
\left(p_{1}, p_{2}\right)=0
$$

or equivalently, that the magnitudes of the real and imaginary patterns are maximal and minimal, respectively, under the side condition (6),

$$
\begin{aligned}
& \left(p_{1}, p_{1}\right)=\left\|p_{1}\right\|^{2}=\max \\
& \left(p_{2}, p_{2}\right)=\left\|p_{2}\right\|^{2}=\min
\end{aligned}
$$

Alternatively, the patterns and associated normal coordinates can be rotated in the complex plane, such that the real and imaginary components of the forcing are statistically orthogonal:

$$
\begin{aligned}
& \left\langle r_{1} r_{2}\right\rangle=0 \\
& \left\langle r_{1} r_{1}\right\rangle=\max \\
& \left\langle r_{2} r_{2}\right\rangle=\min
\end{aligned}
$$

In general, the rotation angles corresponding to the two normalization choices (7)-(9) or (10)-(12) will differ.
In the following discussion we shall choose the complex coordinate axes in accordance with the normalization (7)-(9). This choice has the advantage that it reflects properties of the homogenous system itself, independent of the forcing. It must be kept in mind, however, that the spatially integrated maximum and minimum mean square pattern magnitudes $\left(p_{1}, p_{1}\right)$ and $\left(p_{2}, p_{2}\right)$, respectively, correspond to the actual integrated variance contribution of the patterns only if the excitation is uniform in phase.

If a specific pattern, $p_{1}{ }^{\alpha}=\operatorname{Re}[\exp (i \alpha) p]$, is generated by an initial $\delta(t)$ pulse in some arbitrary direction $\alpha$ in the complex phase plane, the pattern is transformed into the pattern $p_{2}{ }^{\alpha}=$ $\operatorname{Im}[\exp (i \alpha) p]$ after a quarter period $\pi / 2 \omega$. This is replaced by the pattern $-p_{1}{ }^{a}$ after another quarter period, and so on, the amplitudes of both pattern components continually decaying exponentially as $\exp (-t / \tau)$ (cf. Figure 1).

In most applications the state vector $x$ and the eigenvectors $p^{k}$ will represent spatially discretized fields, depending on the spatial coordinate vector $\zeta$. The transformation between the patterns $p_{1}$ and $p_{2}$ during the oscillation can then assume various geometrical waveforms. If $p_{1}(\zeta)=p_{2}\left(\zeta+\zeta_{0}\right)=$ $-p_{1}\left(\zeta+2 \zeta_{0}\right)$, the wave appears as a parallel crested wave of wavelength $L=4\left|\zeta_{0}\right|$, propagating in the $\zeta_{0}$ direction (cf. Figure $2 a$ ). For $p_{1} \gg p_{2}$, the oscillation appears as a standing wave (cf. Figure $2 b$ ). Complex waves of the form considered here occur in the classical harmonic analysis of tides and in the theory of free ocean basin oscillations and are denoted generally as amphidromal (rotational) waves (e.g., Figure $2 c$ ). The modes are often represented in these fields by plots of the local wave amplitude $A(\zeta)=\left[p_{1}(\zeta)^{2}+p_{2}(\zeta)^{2}\right]^{1 / 2}$ and relative phase $\left.\Psi(\zeta)=\tan ^{-1}\left[p_{2}(\zeta) / p_{1}(\zeta)\right)\right]$ (cf. Figure 2, lower panels). The local wave propagation (phase) velocity can then be immediately inferred from the spacing and normal direction of the phase isolines. We shall retain the pattern component representation in the following discussion, since this has some advantages in visualizing the instantaneous anomaly fields.

\subsection{Estimation of the System Matrix}

In the discussion of the normal modes of (1), we have assumed the matrix $B$ to be known. If $B$ cannot be determined from independent theoretical dynamical considerations, it can be estimated by fitting the process (1) to the data. Assuming that the forcing $r(t)$ is uncorrelated with $x(t)$ (or equivalently, by minimizing the forcing) one obtains in the standard manner

$$
B=C_{1} \cdot C_{0}^{-1}
$$

where $C_{1}$ denotes the lag-1 covariance matrix of $x(t)$,

$$
C_{l i j}=\left\langle x_{i}(t+1) x_{j}(t)\right\rangle
$$

and $C_{0}$ denotes the lag- 0 covariance matrix,

$$
C_{0 i j}=\left\langle x_{i}(t) x_{j}(t)\right\rangle
$$

In order to estimate $C_{1}$ and $C_{0}$ in (14) from a finite data set, it is normally useful to reduce the contribution from noisy components in the data by projecting on to a smaller number of large-scale patterns. EOFs are particularly useful in this regard [cf. Preisendorfer et al., 1981; Preisendorfer and Barnett, 1977]. If some time scale is of particular interest, the time series $x(t)$ may also be filtered in time prior to the estimation of $B$. As already noted, we do not address in this paper the 


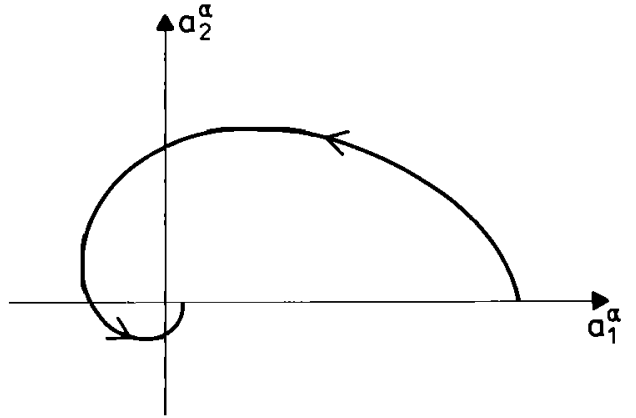

Fig. 1. Temporal development of coefficients $a_{1}{ }^{a}$ and $a_{2}{ }^{x}$ of the complex POP $p^{a}=p_{1}{ }^{a}+i p_{2}{ }^{\alpha}=p \exp (i \alpha)$ excited by an initial $\delta(t)$ pulse acting in an arbitrary direction $\alpha$ in the complex plane.

more general case of the construction of an optimal set of POPs in a space of given dimension $n$, spanned by an unknown set of base vectors, but we assume that the projection of the complete data set on to a smaller set of EOFs removes unwanted noise, while preserving essentially all of the signal we wish to model.

Note in this context that the fitting to the data of a firstorder vector system implies no loss of generality in the formulation of the linear model. A higher-order linear system can always be rewritten as a first-order system by introducing additional variables, defined as the derivatives of the original variables (with a corresponding increase in the dimension of the state vector).

\subsection{Associated Patterns}

After having identified regular oscillatory, standing, or rotary patterns in a multicomponent time series by means of the POP analysis, it is generally useful to search for related signals in further variables not considered in the original POP analysis. This may be carried out using either correlation or composite analysis methods. The correlation method is closer to the basic POP philosophy, since it is based mainly on linear techniques using second-moment stations, while the composite technique has advantages in revealing intermittent, nonlinear, and other non-Gaussian features. In the Gaussian case the two techniques are essentially equivalent.

Associated correlation patterns. The complex associated patterns $q^{k}$ for a vector time series $y(t)$ of dimension $m$, defined for the same time interval as the vector series $x(t)$ used to construct the POPs, are determined in the correlation method as the patterns which yield the best prediction of $y(t)$, using the POP coefficients $a^{k}(t)$ as predictors:

$$
y(t)=\sum_{k} a^{k}(t) q^{k}+s(t)
$$

where the error $s(t)$ is to be minimized. The patterns $q^{k}$ occur in complex conjugate pairs or as real singlets, in the same way as the POPs and the POP coefficients with which they are associated.

Minimizing the expectation value $\left\langle\left(s^{*}, s\right)\right\rangle$ of the integrated square error (the definition of the scalar product $(s, s)$ is immaterial) with respect to variations $\delta q^{k *}$ or $\delta q^{k}$, one obtains in the standard manner

$$
q^{k}=\sum_{l} A_{k l}^{-1}\left\langle a^{l *} y\right\rangle
$$

where

$$
A_{k l}=\left\langle a^{k *} a^{l}\right\rangle
$$

The covariance matrix $A_{k l}$ is in general nondiagonal, since the a)
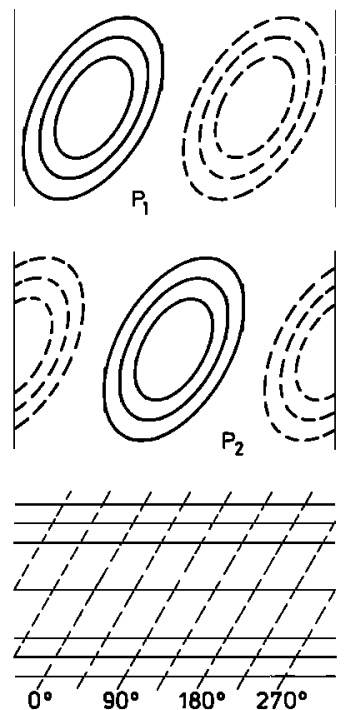

b)
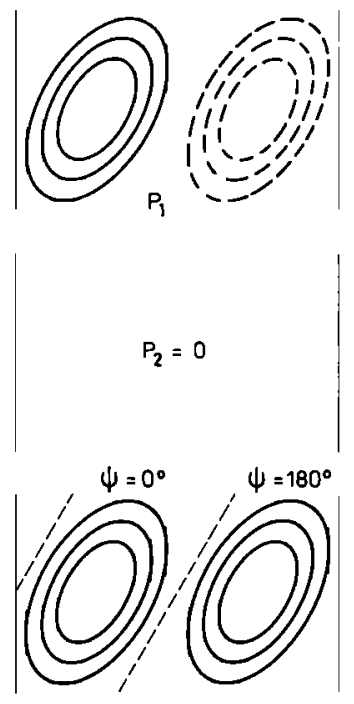

C)

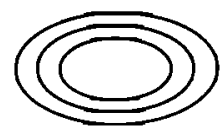

$P_{1}$
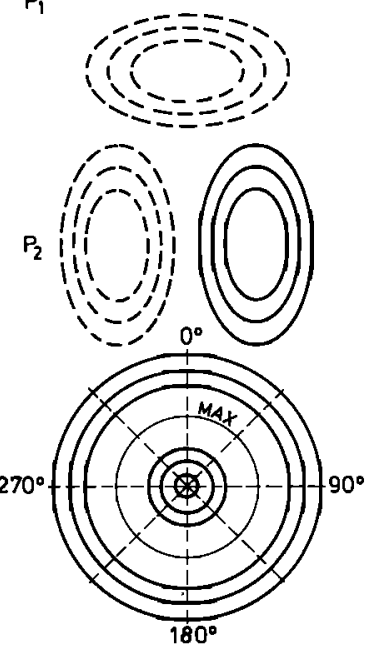

Fig. 2. Different representation of POPs $p_{1}+i p_{2}$ corresponding to (a) a linearly propagating wave, (b) a standing wave, and $(c)$ a purely rotary amphidromic wave. Upper two rows show the representation in terms of real and imaginary parts $p_{1}$ and $p_{2}$. Bottom row shows representation by phases (dashed curve) and amplitudes (solid curve). The POPs in Figures $2 a$ and $2 c$ have the amplitudes shown only if they are generated by a uniform phase forcing function. The amplitude distribution in Figure $2 c$ has minima at the origin and outside the outer circle. The maximum is shown by the light curve. 
POP coefficients are correlated. Thus the associated correlation pattern $q^{k}$ cannot normally be constructed alone from the real and imaginary components of the pattern $\left\langle a^{k *} y\right\rangle$, but consists of a linear superposition of the patterns $\left\langle a^{l *} y\right\rangle$ involving all POP coefficients.

However, in the applications in the present paper we will be concerned with fields containing only a single dominant complex conjugate POP pair,

$$
x(t)=a(t) p+a^{*}(t) p^{*}+n(t)
$$

In this case, (16) reduces to the form

$$
q=\frac{\left\langle|a|^{2}\right\rangle\left\langle a^{*} y\right\rangle-\left\langle a^{* 2}\right\rangle\langle a y\rangle}{\left\langle a^{*} a\right\rangle^{2}-\left\langle a^{2}\right\rangle\left\langle a^{* 2}\right\rangle}
$$

representing a linear combination of the real and imaginary components of $\left\langle a^{*} y\right\rangle\left\langle\left\langle a^{*} a\right\rangle\right.$.

It should be noted that the complex pattern $q$ cannot in general be obtained by a straightforward rotation in the complex plane of the complex pattern $\left\langle a^{*} y\right\rangle$, but consists of some linear combinations of the real and imaginary components of $\left\langle a^{*} y\right\rangle$. In the special case in which the generation of the POP pair is isotropic in the complex plane, however, $\left\langle\left(a^{*}\right)^{2}\right\rangle=$ $\left\langle a^{2}\right\rangle=0$ (since these products are not invariant with respect to rotations), and $q=\left\langle a^{*} y\right\rangle$.

The distinction between a "primary" vector process $x(t)$ which determines the POPs and a "secondary" vector process $y(t)$ for which associated patterns are then derived may be regarded as a little artificial. The systems $x, y$ could have been combined into a single process and the POPs determined simultaneously for the complete system. A greater weighting of the system $x$ relative to $y$, if desired, can be achieved by a suitable choice of the metric defining the quadratic form which is to be minimized. The $y$ components of the resulting POP patterns for the combined analysis can be shown to be closely related to the associated correlation patterns considered here. The main difference between the two methods is that in the combined POP analysis the representation of the field $y$ is optimized with respect to the variance of the time derivative $d y / d t$, rather than $y$ itself. The latter is perhaps preferable if the evolution of the system is assumed to be controlled primarily by the system $x(t)$ and the system $y(t)$ does indeed play only the role of a passive "associated" field.

Associated composite patterns. The construction of composite patterns with respect to externally prescribed indices is a technique which has been widely used in atmospheric and oceanic research. In our application of the method we limit the analysis to a single complex conjugate POP pair.

The input indices are derived from the bivariate time series of the complex coefficient $a(t)$ of the POP pair. Specifically, we take as our two indices the modulus $L(t)$ and the phase $\delta(t)$ of $a(t), a(t)=L(t) \exp [i \delta(t)]$. We divide the complex plane into a set of eight regions (index classes), $K_{1}, K_{2}, \cdots, K_{8}$, representing eight $45^{\circ}$ angular segments for the phase angle $\delta$, beginning with the region $K_{1}:-45^{\circ} / 2<\delta<+45^{\circ} / 2$. Each segment is bounded on the inside by a lower bound limitation on the magnitude of $L$,

$$
L>\langle L\rangle+d \sigma_{L}
$$

where $\sigma_{L}=\left\langle L^{2}-\langle L\rangle^{2}\right\rangle^{1 / 2}$ is the standard deviation of $L$ and we have (arbitrarily) set the coefficient $d=0.8$.

The set of composite patterns for the set of classes are then constructed by averaging over all vectors $y(t)$ for which the associated indices fall into a given class.

For a Gaussian process the resulting composite patterns are independent of the choice of $d$ and are identical to the associated correlation patterns (for the corresponding complex phase angle). The use of eight classes in this case is redundant, since the patterns can be defined by only two components. For a nonlinear, intermittent process, however, the composite patterns differ from the correlation patterns and tend to emphasize the structures associated with stronger events. The composite patterns can also reveal $180^{\circ}$ phase asymmetries and other non-Gaussian features not detected by the correlation method.

\section{The Model Simulation and Fields Selected FOR ANALYSIS}

The data sets used for the POP and associate pattern analysis were taken from the weekly mean time series of standard atmospheric variables generated in a 10 -year climate simulation with the ECMWF T21 GCM. The numerical experiment was carried out jointly by the Meteorological Institute of the University of Hamburg and the Max Planck Institute of Meteorology in Hamburg. The boundary conditions corresponded to the climatological annual cycle, with no interannual anomaly forcing. The simulation reproduces most of the basic features of the general circulation of the atmosphere [cf. Fischer, 1987].

The POP analysis was carried out for the $200-\mathrm{hPa}$ velocity potential field along the equator, averaged meridionally between $5^{\circ} \mathrm{N}$ and $5^{\circ} \mathrm{S}$. The velocity potential was chosen, rather than the wind components or the outgoing long-wave radiation, for example, since the divergent component of the wind, weighted toward small wave numbers through the use of the potential function, emphasises the Kelvin wave structure which we anticipate in this region [Lorenc, 1984]. The restriction to the equatorial band was similarly motivated (and also limited the numeral scope of the computations).

The time series $y(t)$ for which the associated patterns were computed cover the global tropics from $30^{\circ} \mathrm{N}$ to $30^{\circ} \mathrm{S}$, in contrast to the equatorial time series $x(t)$ used to define the POPs.

Prior to the POP analysis, a standard cross-spectral analysis was carried out for a few typical locations. Figures $3 a-3 c$ show the variance, coherence, and phase spectra for a pair of locations in the west Pacific $\left(150^{\circ} \mathrm{E}\right)$ and the east Pacific $\left(100^{\circ} \mathrm{W}\right)$. Because of the stronger convective activity in the western Pacific, the 200-hPa variance is almost an order of magnitude greater in the west Pacific (Figure $3 a$, solid curve) than in the east Pacific (dashed curve). Both spectra exhibit dominant annual and semiannual cycles, while the interannual variance is small, presumably because the prescribed climatological sea surface temperature (SST) contained no variability in these time scales.

The variance distribution on intraseasonal time scales appears white. A 30 - to 60-day wave is not immediately discernible as a peak in the variance spectra. However, the increased levels of the coherence spectra (Figure $3 b$ ) for periods shorter than 60 days indicate that a signal characterized by a large spatial scale exists in this frequency band. The out-of-phase relationship between $150^{\circ} \mathrm{E}$ and $100^{\circ} \mathrm{W}$ (Figure $3 c$, heavy curve) implies a characteristic half-wavelength scale of order $100^{\circ}$ longitude, while the $180^{\circ}$ phase difference between the 

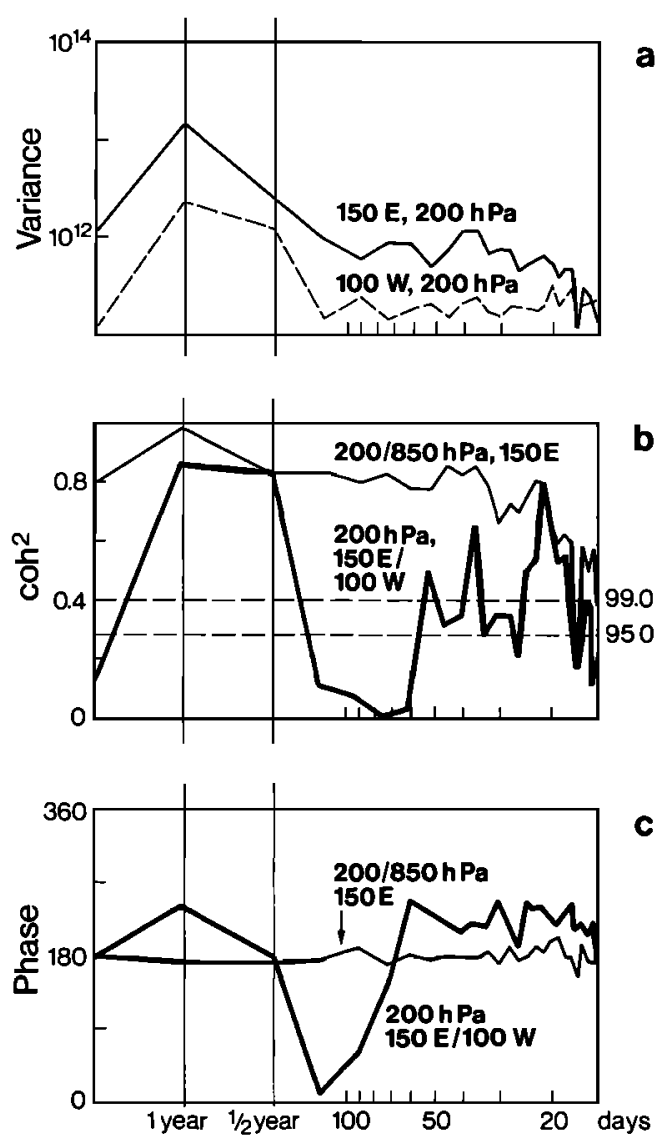

F1g. 3. Cross-spectral analysis of equatorial velocity potential. (a) Upper tropospheric variance spectra at $150^{\circ} \mathrm{E}$ and $100^{\circ} \mathrm{W}$. (b) Coherence squared between upper and lower tropospheric velocity potential at $150^{\circ} \mathrm{E}$ (light curve) and between $200-\mathrm{hPa}$ velocity potential at $150^{\circ} \mathrm{E}$ and at $100^{\circ} \mathrm{W}$ (heavy curve). The dashed curves represent the 95 and $99 \%$ confidence limits. (c) Phase spectra corresponding to Figure $3 b$.

upper and lower level velocity potential (Figure 3c, light curve) is suggestive of deep convection processes.

Since we were interested in this study in the time scale of a few weeks, all time series were high-pass filtered to suppress the dominant seasonal cycle. The filter function was set to 1 for time scales of 2-8 weeks and to 0 for time scales larger than 10 weeks, with the transition region of 8-10 weeks being represented by a smooth (quarter-wave) cosine function (waves shorter than 2 weeks are not resolved by the weekly data series). This filter proved relatively effective in removing secondary maxima in the representation of singular events, such as a pulse, in the time domain. The results are relatively insensitive to the details of the filter chosen.

\section{Results}

\subsection{POP Analysis}

Before carrying out the POP analysis of the high-pass filtered equatorial velocity potential at $200 \mathrm{hPa}$, the data were expanded in an EOF series. The expansion was truncated after 10 terms. The truncated series retained more than $95 \%$ of the (high-pass filtered) variance. The purpose of the EOF expansion was to remove small-scale noise and to reduce the dimension of the system. Details of the EOF expansion, in- cluding questions such as the significance and degeneracy of the EOF patterns, are not relevant in the present context, since we are concerned only with the net space spanned by the reduced representation.

All of the POPs of the reduced 10-dimensional system were complex, i.e., we obtained five pairs of complex patterns $p^{k}=$ $p_{1}{ }^{k}+i p_{2}{ }^{k}, k=1,3,5,7$, and $9(k=2,4,6,8$, and 10 refer to the complex conjugate patterns).

All but the first pair of POPs have characteristic $e$-folding times less than 1 week (cf. Table 1). The variance of the coefficients of this pair is also the largest: $\left\langle a_{1}(t)^{2}\right\rangle=\left(12.95 \times 10^{6}\right.$ $\left.\mathrm{m}^{2} \mathrm{~s}^{-1}\right)^{2}$ and $\left\langle a_{2}(t)^{2}\right\rangle=\left(14.31 \times 10^{6} \mathrm{~m}^{2} \mathrm{~s}^{-1}\right)^{2}$, where the real and imaginary components of the patterns are orthogonal and are normalized, such that

$$
\left\|p_{1}\right\|^{2}+\left\|p_{2}\right\|^{2}=\sum_{j=1}^{n}\left[p_{1}{ }^{2}(j)+{p_{2}}^{2}(j)\right]=1
$$

the sum being taken over all grid points $j$ along the equator. The first POP pair explains $60 \%$ of the total variance in the spectral band for 3-10 weeks. The ratios of the real and the imaginary components are $\left\|p_{1}\right\|^{2} /\left\|p_{2}\right\|^{2}=1.6$. Thus the generation is fairly isotropic (to within $10 \%$ ), but the magnitudes of the two patterns differ significantly (by $60 \%$ ). We shall consider only this pair $p_{1}, p_{2}$ in the following (and omit the index $k=1$ ).

The orthogonal patterns $p_{1}$ and $p_{2}$, normalized according to (7)-(9) and (21) are shown in Figure $4 a$. Both exhibit a similar wave number 1 spatial structure, with a phase shift of order $90^{\circ}$, corresponding to an eastward propagating disturbance. However, the patterns are not exactly sinusoidal, as required for pure propagation without distortion. In a period of 24 days the patterns propagate once around the globe but, because of the relatively short $e$-folding decay time of 10 days, individual wave events are damped out before the disturbances have propagated more than half a wavelength.

The gradual transition $p_{1} \rightarrow p_{2} \rightarrow-p_{1} \rightarrow-p_{2} \rightarrow p_{1}$ may be identified with an eastward displacement of the whole pattern. The maximum, which is initially located slightly to the west of the dateline, moves smoothly to a position slightly east of the dateline within about 6 days, when $p_{2}$ has become dominant. After 12 days a $-p_{1}$ pattern has evolved, with a maximum at about $60^{\circ} \mathrm{W}$. Finally, 18 days after starting our hypothetical cycle, a $p_{2}$ pattern is found, with a maximum at about $90^{\circ} \mathrm{E}$, but the entire system has been attenuated at this time by the factor $\exp (-18 / 10)=1 / 6$.

For interest we also show in Figure $4 b$ the patterns obtained by the alternative normalization (10)-(12). These patterns are no longer spatially orthogonal, but are forced by statistically orthogonal noise. The standard deviation of the

TABLE 1. Characteristics of POPs of High-Pass Filtered Equatorial Velocity Potential at $200 \mathrm{hPa}$

\begin{tabular}{ccc}
\hline $\begin{array}{c}\text { Number of } \\
k\end{array}$ & $\begin{array}{c}T_{h}, \\
\text { weeks }\end{array}$ & $\begin{array}{c}\tau_{h}, \\
\text { weeks }\end{array}$ \\
\hline $1 / 2$ & 3.4 & 1.5 \\
$3 / 4$ & 2.1 & 0.8 \\
$5 / 6$ & 2.1 & 0.6 \\
$7 / 8$ & 2.4 & 0.7 \\
$9 / 10$ & 2.8 & 0.5 \\
\hline
\end{tabular}

$T_{h}$, period; $\tau_{h}=e$-folding time. 
a)

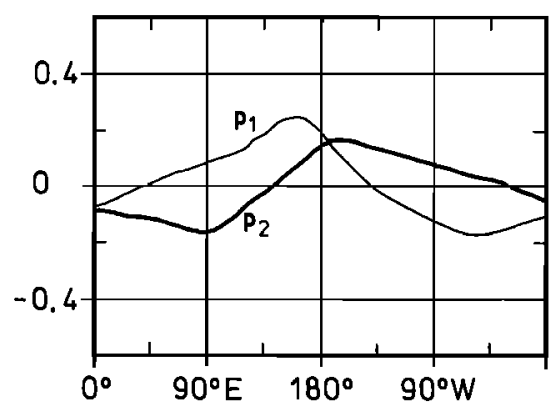

b)

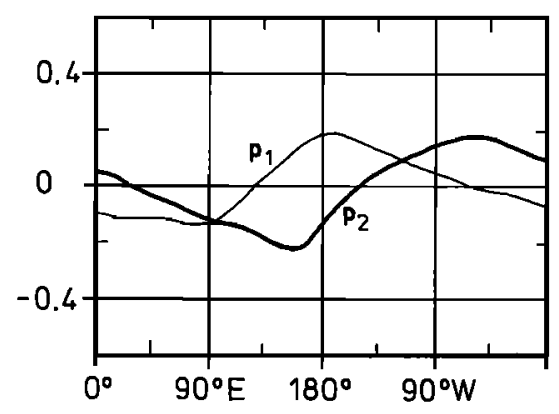

Fig. 4. Spatial structure of first POP pair, $p_{1}+i p_{2}$. The real part, $p_{1}$, is denoted by the light curve, the imaginary component, $p_{2}$, by the heavy curve. (a) Normalization according to equations (7) -(9) with orthogonal patterns $p_{1}$ and $p_{2}$. (b) Normalization according to equations (10)-(12) with statistical independent forcing $r_{2}$ and $r_{2}$.

noise acting on $p_{1}$ and $p_{2}$ is $18.6 \times 10^{6} \mathrm{~m}^{2} \mathrm{~s}^{-1}$ and $17.3 \times 10^{6}$ $\mathrm{m}^{2} \mathrm{~s}^{-1}$, respectively. The two representations differ only with respect to the choice of the reference phase $\theta$ in the complex plane. The phase difference is $77^{\circ}$. The larger pattern $p_{1}$ is not strongly affected by the transformation, but the smaller pattern $p_{2}$ clearly receives a significant admixture of $p_{1}$ in transforming to the orthogonal forcing reference system. In the following, we limit ourselves to the orthogonal nomalization (7)-(9).

The theoretical forced damped oscillator properties of the complex POP coefficient $a(t)=a_{1}(t)+i a_{2}(t)$ are confirmed by the cross-spectral analysis of the time series $a_{1}(t)$ and $a_{2}(t)$ shown in Figure 5 . The spectra show an approximately equal distribution of variance (in accordance with the approximately isotropic forcing), a constant phase difference of $-90^{\circ}$, and a significant coherence throughout the period range between 3 and 8 weeks. The POP pair $p_{1} / p_{2}$ therefore appears to be representative of the intraseasonal variability in a rather broad frequency band.

Another way of testing whether a rotation between the patterns in the complex plane is really taking place is to compute the histogram of the changes $\Delta \alpha$ in phase angle $\alpha(t)=\tan ^{-1}$ $\left(a_{1} / a_{2}\right)$ of the complex coefficient $a=a_{1}+i a_{2}$ between one time level and the next, $\Delta \alpha=\alpha(t+1)-\alpha(t)$. Figure 6 shows the resulting distribution of $\Delta \alpha$, based on a discretization of the phase angle of $45^{\circ}$. (This discretization corresponds to the index classes used on the composite associate pattern analysis discussed in section 2.4 , and the transition probabilities were actually computed from the transitions between index classes.) For a time discretization $\Delta t=1$ week, the maximum transi-
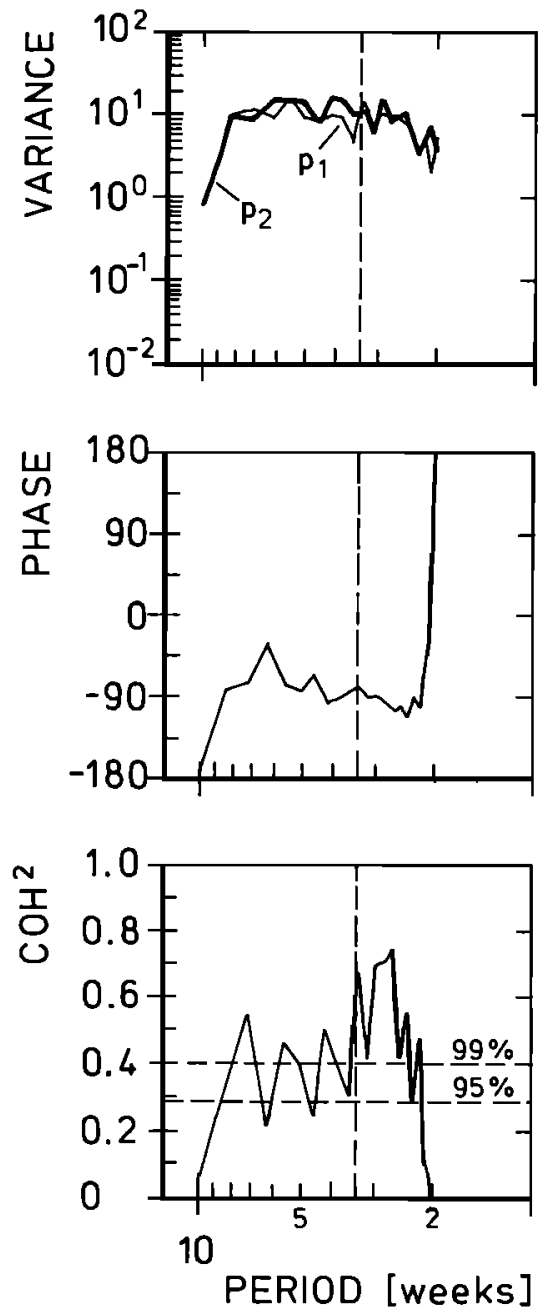

Fig. 5. Cross-spectral analysis of POP coefficient time series $a_{1}(t)$ and $a_{2}(t)$ for the POPs $p_{1}$ and $p_{2}$, shown in Figure 4a. The lowfrequency cutoff at about 10 weeks is due to filtering. The vertical dashed line indicates the POP oscillation period of 24 days.

tion probability in the bin $\Delta \alpha=90^{\circ}$ corresponds to a rate of change of the phase angle of $\omega=90^{\circ} / 1$ week $=360^{\circ} / 4$ weeks. Allowing for the rather coarse resolution and the spread of the distribution (which actually yields a lower mean period than the period of the maximal probability bin), the period of 4 weeks estimated in this manner is consistent with the period of 3.4 weeks listed in Table 1 . The spread of the distribution is also consistent with the relatively large damping of the oscillation, which corresponds to a rather broad spectral distribution (cf. Figure 5). It is encouraging that Figure 6 shows a reasonably smooth distribution, with no outliers at very high or low values, which would indicate a breakdown of the model for certain events.

For comparison, we also analyzed the data with the complex EOF technique [Horel, 1984] and obtained very similar patterns (not shown).

Since the variance of the 30 - to 60 -day velocity potential waves observed in the atmosphere is known to be concentrated in zonal wave number 1 , we also performed a standard wave number-frequency analysis. Almost all of the variance was indeed concentrated at zonal wave number 1 . Using Hayashi's [1982] technique for the decomposition into stand- 


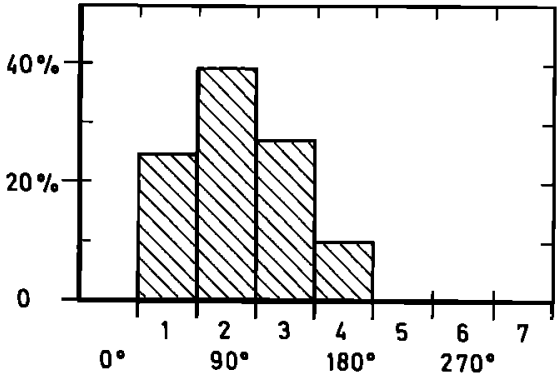

Fig. 6. Histogram of phase changes $\Delta \alpha$ occurring in the time in crement $\Delta t=1$ week (constructed from transitions between $45^{\circ}$ discrete phase angle index classes).

ing and traveling waves, it was found that the eastward traveling waves clearly dominate over the standing and westward traveling waves on time scales of a lew weeks (Figure 7). (Hayashi's technique must be applied with some caution, since the $\omega, k$ Fourier components cannot generally be linearly transformed into statistically orthogonal standing and traveling wave components [cf. Luksch et al., 1987].

Eastward propagating waves can also be recognized visually in the high-pass filtered time-longitude (Hovmeller) diagram (Figure $8 a$ ) showing the evolution of the $200-\mathrm{hPa}$ velocity potential from April through December during the first model year. Two episodes of relatively strong wave activity can be observed in this period, beginning in August and October, respectively. The origin of the waves is located in the Indonesian region, where the variability generally is largest. Following a fairly long period of irregular behavior (AprilJuly), the first event, consisting of a strong negative anomaly over Indonesia associated with upper level outflow and enhanced convection, develops in the first half of August. During the following month, the disturbance travels eastward across the Pacific, while decreasing in amplitude.

The second episode occurs in late October, with somewhat less intensity. After crossing the Pacific the wave appears to receive a second forcing pulse over South America and is thus able to propagate around the globe back into the original forcing region, where it is reinforced again at the end of November. The wave generation then appears to be interrupted for a time, but it recovers again late in December, when a disturbance again crosses the Pacific.

Figure $8 b$ shows the corresponding Hovmøller diagram for the filtered field containing only the contributions from the first POP pair. It is evident that the events shown in Figure $8 a$ do actually coincide with an amplification of the first POP pair.

An inspection of the Hovmøller diagram for the complete 10-year time series (not shown) indicates that the examples discussed are representative of the overall model behavior, although different events never evolve identically. In general, the occurrence of these Kelvin-type waves seems to be random, rather than coupled to certain seasons, as found also by Madden [1986] and Knutson and Weickmann [1987] in observations.

In general, the properties of the waves occurring in the T21 model, as inferred from the equatorial $200-\mathrm{hPa}$ velocity potential, appear to be qualitatively similar to those observed in the 30- to 60 -day period range. In subsection 4.2 , we consider the associated patterns of additional fields, thereby obtaining further insight into the structure of the model's 30- to 60-day wave.

\subsection{Associated Patterns of Tropical Upper Air Parameters}

Using the time series of the complex POP coefficient $a(t)$, associated correlation and composite patterns were constructed for the 200- and $850-\mathrm{hPa}$ velocity potential $\mathrm{X}$ and the 200-hPa stream function $\Psi$ for the tropical region between $30^{\circ} \mathrm{N}$ and $30^{\circ} \mathrm{S}$.

The associated composite patterns of the 200-hPa tropical velocity potential, shown in Figure 9 as a pseudo-Hovmøller diagram, represent means of eight to 12 events. They reproduce the POPs along the equator (as, of course, they must, since the POPs themselves are derived from the $200-\mathrm{hPa}$ velocity potential along the equator). Away from equator the amplitude of the associated pattern decreases. The largely zonal structures are probably the result of annual averaging; individual seasons would presumably show stronger meridional features [Madden, 1986; Knutson and Weickmann, 1987].

The maximum in the category $K_{1}$ composite $\left(a_{1}\right.$ maximum positive; $a_{2}$ close to zero) is $4-5 \times 10^{6} \mathrm{~m}^{2} \mathrm{~s}^{-1}$, but the minimum in the $180^{\circ}$ phase-shifted $K_{5}$ composite ( $a_{1}$ maximum, negative; $a_{2}$ close to zero) is $-7 \times 10^{6} \mathrm{~m}^{2} \mathrm{~s}^{-1}$. The asymmetry indicates that the CISK-supported upper air divergence is stronger than the upper air convergence associated with subsidence.

In Figure $10 a$ the longitudinal location of the equatorial maxima and minima in the series of $K_{\imath}$ composites (Figure 9) is shown as a function of category index $i$. From this diagram, propagation velocities are derived and are shown in Figure $10 b$ as dashed curves. The disturbances travel rapidly, at more than $30 \mathrm{~m} \mathrm{~s}^{-1}$ over the eastern Pacific, the Atlantic, and the Indian Oceans. Over the western Pacific and Indonesia, South America, and Africa, the propagation velocities are generally less than $15 \mathrm{~m} \mathrm{~s}^{-1}$. There is a tendency for larger velocities to occur in the western hemisphere. This is consistent with the zonal variation of the phase velocity found by Madden and Julian [1972] in station data and by Knutson et al. [1986] in an observed $250-\mathrm{hPa}$ tropical zonal wind time series. The propagation velocities in their analyses varied between $6 \mathrm{~m}$ $\mathrm{s}^{-1}\left(60^{\circ}-160^{\circ} \mathrm{E}\right)$ and $15 \mathrm{~m} \mathrm{~s}^{-1}\left(160^{\circ} \mathrm{E}-0^{\circ}\right)$. This effect may be partly due to a Doppler shift caused by the background flow, which is westerly on the average but easterly in the Indian Ocean and in the Western Pacific.

An interesting feature is the amplification over Indonesia, which is seen to occur simultaneously with a decrease in the

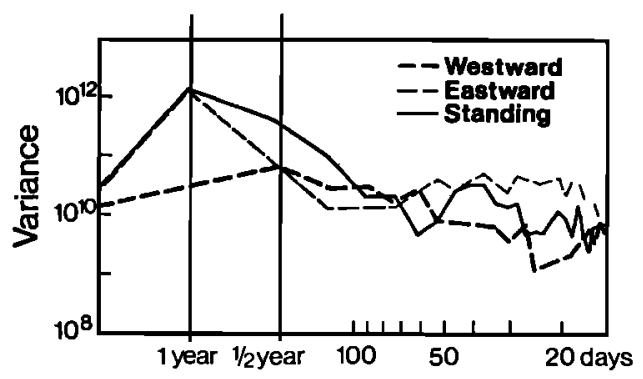

Fig. 7. Variance spectra attributed to westward and eastward travelıng waves and standing waves for zonal wave number 1. 

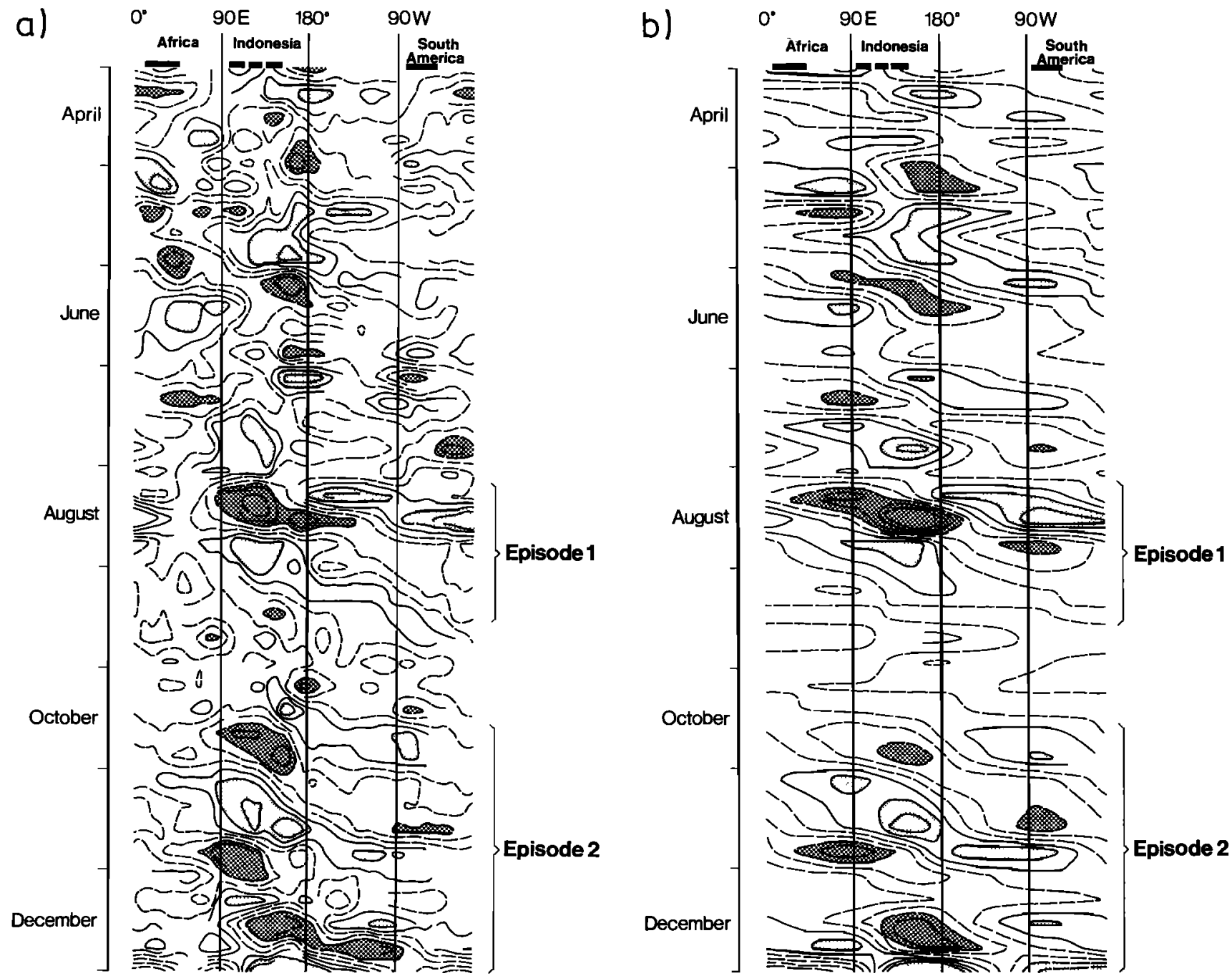

Fig. 8. Hovmøller diagram of equatorial $200-\mathrm{hPa}$ velocity potential in the spectral band 2-8 weeks for the first year of integration. Dark shaded areas indicate negative events smaller than $-3 \times 10^{6} \mathrm{~m}^{2} \mathrm{~s}^{-1}$; light shaded areas indicate positive events greater than $3 \times 10^{6} \mathrm{~m}^{2} \mathrm{~s}^{-1}$. (a) Original data, i.e., $x(t)$. (b) Projection of original data onto first POP pair, i.e., $a_{1}(t) p_{1}+a_{2}(t) p_{2}$.

propagation velocity (minimum values of less than $10 \mathrm{~m} \mathrm{~s}^{-1}$; see Figure 10b). This area has been identified as a region of maximum heating in the annual mean [Bruns and von Storch, 1986]. The present model result is consistent with results derived from observations by Madden and Julian [1972] and Madden [1986].

Figure 11 shows the two associated correlation patterns $q_{1} \mathrm{X}, q_{2} \mathrm{X}$ for the $200-\mathrm{hPa}$ velocity potential $\mathrm{X}$. They exhibit essentially the same propagation features already seen in the composite sequence (Figure 9). The patterns shown in Figures 11,12 , and 15-16 have been multiplied with the standard deviations of the POP coefficients $a_{1}(t)$ and $a_{2}(t)$, respectively, in order to show not only the patterns, but also the characteristic amplitude. Since the composites are constructed from episodes for which the coefficient amplitudes $L(t)$ exceed the mean by at least 0.8 standard deviations (cf. section 2.4 ), one obtains systematically larger magnitudes for the composite patterns than for the associated correlation patterns, which are calculated from all states. The ratio of the magnitudes of the associated and correlation patterns is of order 2, which corresponds rather closely to the ratio of the index class mean amplitude to the true mean amplitude, as expected.

The analysis of the $850-\mathrm{hPa}$ velocity potential yields the same associated correlation patterns (not shown), but with opposite signs. In particular, no significant vertical tilt of the velocity potential is found, so that the low- and high-level divergent flows appear almost exactly out of phase.

The maximum values of the associated velocity potential correlation patterns are of order $1 \times 10^{6} \mathrm{~m}^{2} \mathrm{~s}^{-1}$ at both levels. The explained variance is generally $40 \%$ or somewhat smaller, with maximum values near $65 \%$ at the equator. The maximum anomalies are of order $7 \times 10^{6} \mathrm{~m}^{2} \mathrm{~s}^{-1}$, which is significantly larger than the maximal interannual anomalies of the model, of about $4 \times 10^{6} \mathrm{~m}^{2} \mathrm{~s}^{-1}$ [Bruns and von Storch, 1986].

Lau and Lau [1986], and Knutson and Weickmann [1987] found patterns similar to the associated patterns discussed here in Geophysical Fluid Dynamics Laboratory GCM simulations and in the National Meteorological Center analyses, respectively, using an EOF/composite analysis method. 

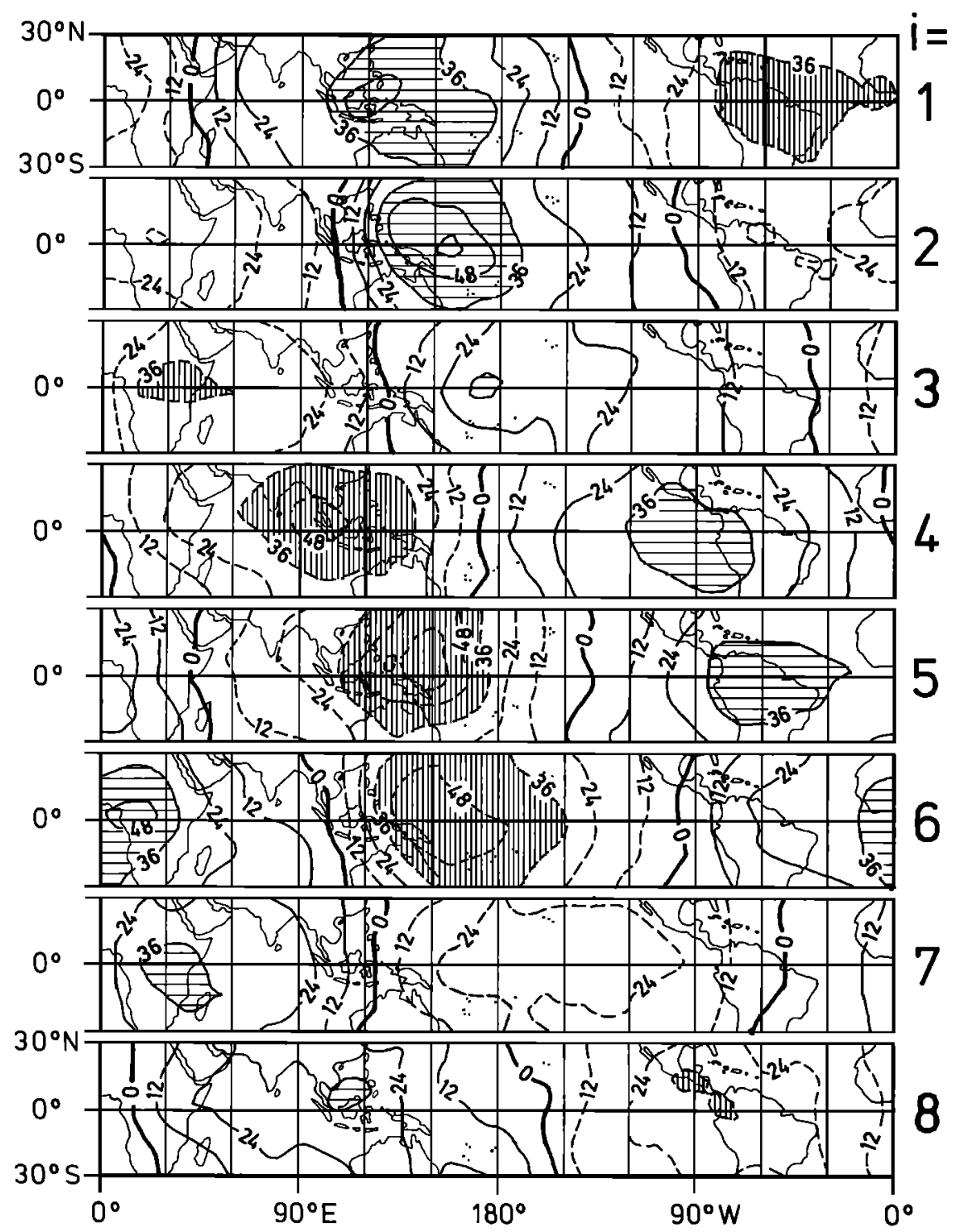

Fig. 9. Composites $K_{t}$ of tropical 200 -hPa velocity potential. The $i$ th panel (from top) shows composite $K_{\imath}$. The ordering of the composites yields a composite Hovmoller diagram, since increasing phase angle corresponds to increasing time. Units are $10 \mathrm{~m}^{2} \mathrm{~s}^{-1}$.

In the case of the $200-\mathrm{hPa}$ stream function $\Psi$, the sequence of the associated composite patterns (not shown) and correlation patterns $q_{1}{ }^{\Psi}, q_{1}{ }^{\Psi}$ (Figure 12 ) are very similar. Zonally, the patterns are dominated by a wave number 1 scale flow, which straddles the equator symmetrically. The two patterns are almost perfectly phase shifted and have the same "quadrupole" form. The velocity field associated with this pattern is almost completely zonal, with no cross-equatorial flow. If the extremes of the velocity potential in Figure 11 are identified with the extremes of the anomalous forcing of the equatorial troposphere, we find phase reversals of the stream function at the maxima and minima of the anomalous equatorial forcing. This is in accordance with the relationship between the velocity potential and the stream function of an equatorially trapped Kelvin wave [cf. Hendon, 1986]. The $q_{1}{ }^{\Psi}$ pattern strongly resembles the schematic diagram given by Weickmann et al. [1985, p. 952].

The explained variance is generally less than $5 \%$ on the equator and reaches maximum values of $10-20 \%$ at $15^{\circ} \mathrm{N}$ and $15^{\circ} \mathrm{S}$.

We note again that the almost perfect symmetry of the patterns found in our analyses about the equator is probably due to the fact that we have not distinguished between seasons. Asymmetries may be expected to occur in different seasons [Madden, 1986; Knutson and Weickmann, 1987], but these will tend to average out in forming the annual mean.

\subsection{Associated Correlation Patterns of Surface Data}

It has been speculated that the 30- to 60-day wave may be the random process triggering the development of the warm and cold events of the Southern Oscillation. It is thus of particular interest to investigate further the properties of the POP waves with respect to the associated surface wind, sea level pressure, and precipitation patterns. For the sake of brevity 

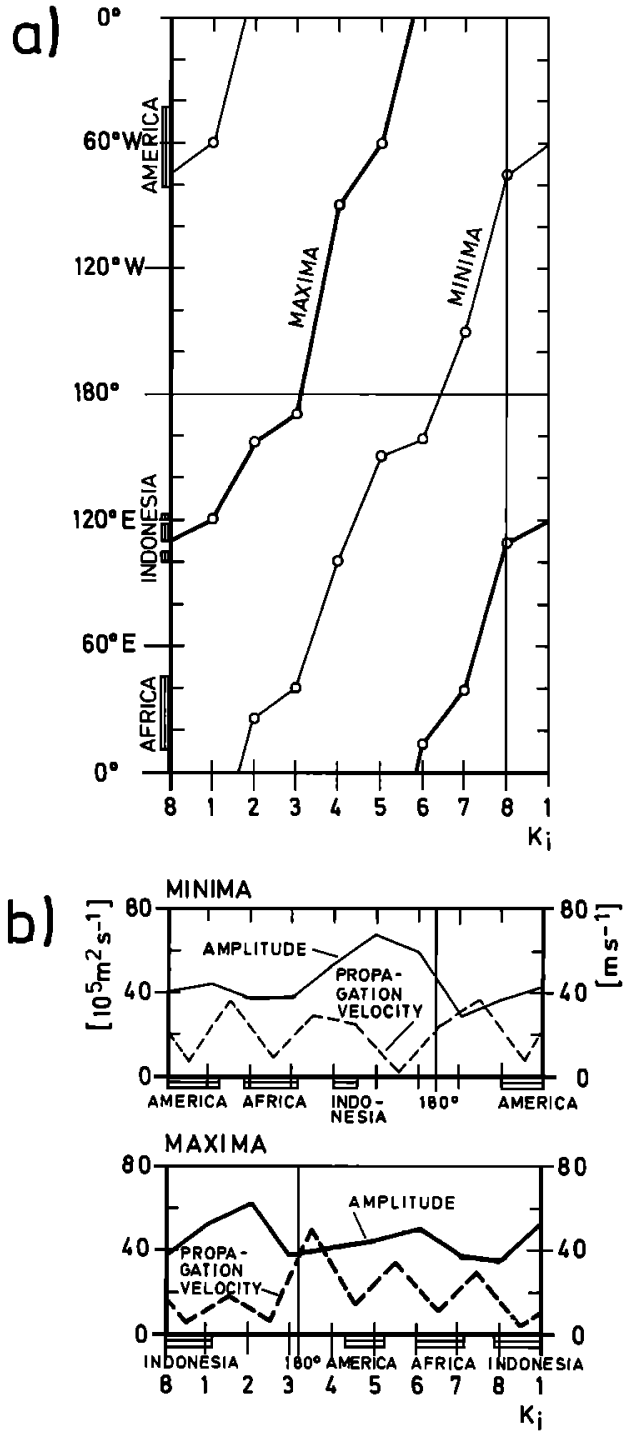

Fig. 10. (a) Propagation along the equator of maxima and minima of $K_{1}$ composites shown in Figure 9. Horizontal axis indicates index $i$ of categories $K_{i}$; vertical axis shows longitude. (b) Phase velocity (in meters per second) and strength of (top) minima and (bottom) maxima $\left(10^{6} \mathrm{~m}^{2} \mathrm{~s}^{-1}\right)$ as function of class $K_{\text {. }}$.

we limit the discussion in this section to the associated correlation patterns only.

Sea level pressure. The associated correlation patterns of sea level pressure (SLP) $q_{1}{ }^{\text {SLP }}, q_{2}{ }^{\text {SLP }}$ shown in Figure 13 are strongly reminiscent of Matsuno's [1966] and Gill's [1980] simplified linear solutions of the response of the tropical atmosphere's pressure to stationary diabatic heating. The response pattern consists of a downstream equatorial trough (Kelvin wave response) and two off-equatorial cyclones (Rossby wave response) slightly to the west of the maximum of the forcing anomaly (Figure 11). Cooling generates a pattern of opposite sign. The fact that equatorial SLP leads the subtropical SLP at the same longitude has been noted previously in atmospheric observations by Madden and Julian [1972] and in GCM data by Lau and Lau [1986].

The associated correlation patterns of SLP and the $200-\mathrm{hPa}$ stream function appear quite different. However, they are consistent with an overall baroclinic behavior. In the northern hemisphere, where the Coriolis parameter $f$ is positive, negative SLP anomalies are found together with positive stream function anomalies, while in the southern hemisphere, where $f$ is negative, the two types of anomalies have the same sign. On the equator, where $f$ vanishes, the direction of the low-level flow is opposite to that at the upper level.

The GCM 30- to 60-day wave is associated with a highfrequency Southern Oscillation index, as shown in Figure 14. During strong episodes (identified in the composites), SO indices of order $-1 \mathrm{hPa}$ can be found. This may be compared with a winter mean Southern Oscillation anomaly of $-2 \mathrm{hPa}$, which was simulated by the GCM when forced by the observed extreme 1982/1983 El Niño SST anomaly in the equatorial Pacific (E. Kirk, personal communication, 1987).

The explained variance has maximum values along the equator ranging from $8 \%$ east of Indonesia to $16 \%$ for East Africa. Off the equator the explained variance is generally less than $4 \%$.

Surface wind. A significant signal is found in the associated correlation pattern $q_{1}{ }^{\mu}, q_{2}{ }^{\mu}$ of the zonal wind component (Figure 15) but not in the meridional component (not shown). Although seen less clearly in the POP $p_{1}$ pattern than in the $p_{2}$ pattern, the centers of the maximal surface zonal wind anomalies (maximum values $\sim 0.5 \mathrm{~m} \mathrm{~s}^{-1}$ ) appear to be connected with maximal gradients of the velocity potential anomalies. (Maximum values in the composite patterns are typically $1 \mathrm{~m} \mathrm{~s}^{-1}$ ).

The zonal wind component pattern is generally consistent with the Gill-type response described earlier, with surface westerlies east of the forcing region in the case of cooling. However, the detailed structure of the GCM $10-\mathrm{m}$ wind field exhibits only a weak similarity with the theoretical wind field. This is perhaps to be expected, since the boundary layer $10-\mathrm{m}$

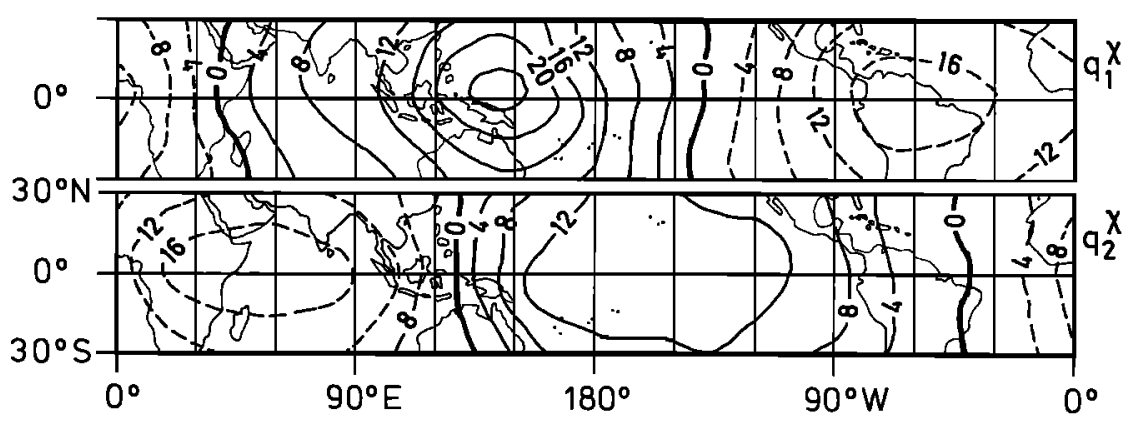

Fig. 11. (Top) Associated patterns $q_{1}{ }^{\mathrm{X}}$ and (bottom) $p_{2}{ }^{\mathrm{X}}$ of $200-\mathrm{hPa}$ tropical velocity potential. The patterns are normalized by the standard deviation of $a_{1}(t)$ and $a_{2}(t)$, respectively, and are in units of $10^{5} \mathrm{~m}^{2} \mathrm{~s}^{-1}$. The pattern $p_{1} \mathrm{X}$ corresponds to the $K_{1}$ (and $K_{5}$ ) composite (of Figure 9), the pattern $q_{2}{ }^{x}$ corresponds to the $K_{3}$ (and $K_{7}$ ) composite. 


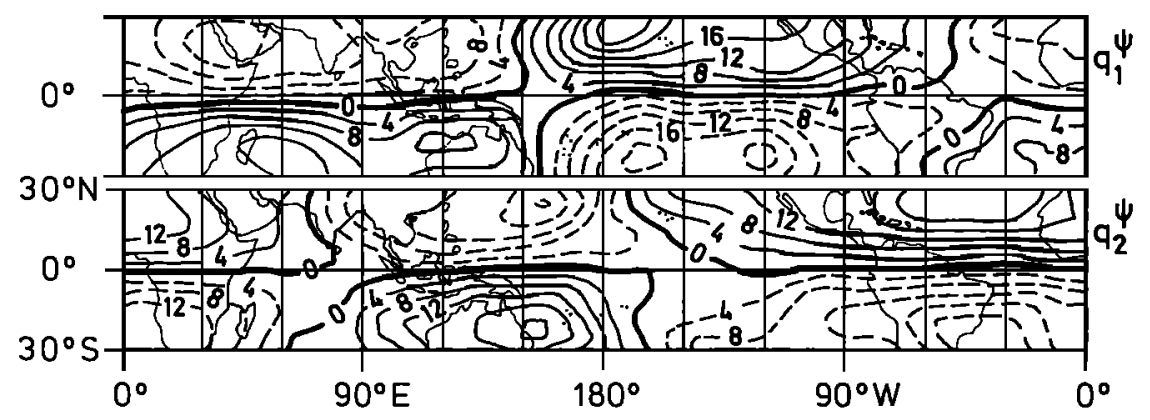

Fig. 12. Associated patterns $q_{1}{ }^{\Psi}$ and $q_{2}{ }^{\Psi}$ for 200 -hPa stream function, with same normalization and units as Figure 11.

wind is strongly influenced by local properties, such as the land-sea distribution and orography.

The explained variance is about $4-8 \%$ along the equator in the Pacific for the zonal wind but no more than $1 \%$ for the meridional component. The fact that we have not stratified the data with respect to season may have been a contributing factor to the rather low explained variance value [cf. Madden, 1986].

Precipitation. The associated correlation patterns of convective rainfall are noisier still than the surface wind patterns, and the explained variance is correspondingly small ( $2 \%)$. Presumably, this is related to the high-frequency variability and the small spatial scale of tropical precipitation.

Nevertheless, the associated correlation patterns $q_{1}{ }^{p r}$ and $q_{2}{ }^{p r}$, shown in Figure 16, appear reasonably well defined. The minimum and maximum of the velocity potential pattern $p_{1}$ (Figure $4 a$ ) are associated with a maximum and a minimum of the rainfall pattern $q_{1}{ }^{p r}$. The signal is significantly stronger over Indonesia than over eastern South America. In the pattern $p_{2}$ (Figure $4 a$ ), however, the extremes of the velocity potential anomaly are not at exactly the same positions as the extremes for the rainfall pattern $q_{2}{ }^{p r}$. Positive velocity potential anomalies are found east of $140^{\circ} \mathrm{E}$, with maximum values east of the dateline, while negative anomalies are largest at $90^{\circ} \mathrm{E}$. The associated patterns of precipitation, on the other hand, are confined to the eastern hemisphere, from about $60^{\circ}$ to $180^{\circ} \mathrm{E}$, with maximum negative values of $6 \mathrm{~mm}^{\mathrm{day}}{ }^{-1} \mathrm{oc}-$ curring in the region $150^{\circ}-180^{\circ} \mathrm{E}$ and maximum positive values of $4 \mathrm{~mm} \mathrm{day}^{-1}$ at $110^{\circ} \mathrm{E}$

A comparison of the GCM annual mean rainfall distribution with the associated correlation velocity potential and precipitation patterns reveals that the strongest signals occur in the Indonesia-Western Pacific area, where maximum rainfall also occurs in the GCM climatology.

Interestingly, the slowest eastward migration (Figure 10) takes place in regions where the extreme values of the precipitation and velocity potential patterns are in the same position, while the propagation is relatively fast in regions where they are displaced relatively to each other. An examination of the associated $q_{2}$ patterns of sea level pressure (Figure 13) and zonal surface wind (Figure 15) reveals significant anomalies at latitudes $120^{\circ}-180^{\circ} \mathrm{E}$ which are also not related in phase to the velocity potential $p_{2}$ pattern. Knutson and Weickmann [1987] found a similar dependence of the relationship between the tropical heating and velocity potential in their investigations of outgoing long-wave radiation using observed data.

\section{Conclusions}

Intraseasonal oscillations found in the tropical ECMWF T21 GCM atmosphere have been analyzed by a new technique using principal oscillation patterns. The POP analysis identifies an eastward traveling, damped wave number 1 type
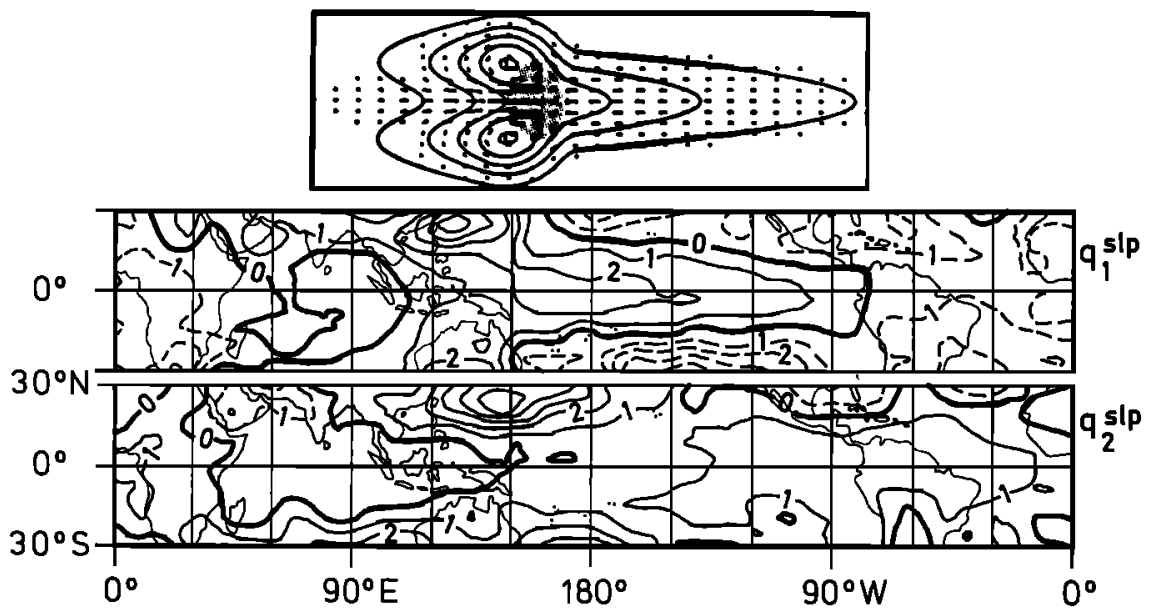

Fig. 13. (Top) Response of a linearized tropical atmosphere to equatorial forcing. The forcing area is shaded [from Gill, 1980]. (Bottom two panels) Associated patterns $q_{1}{ }^{\text {sLP }}$ and $q_{2}{ }^{\text {SLP }}$ for sea level pressure. Normalization as in Figure 11, with units of hectopascals. Note that the signs in the top and bottom two diagrams are reversed. 


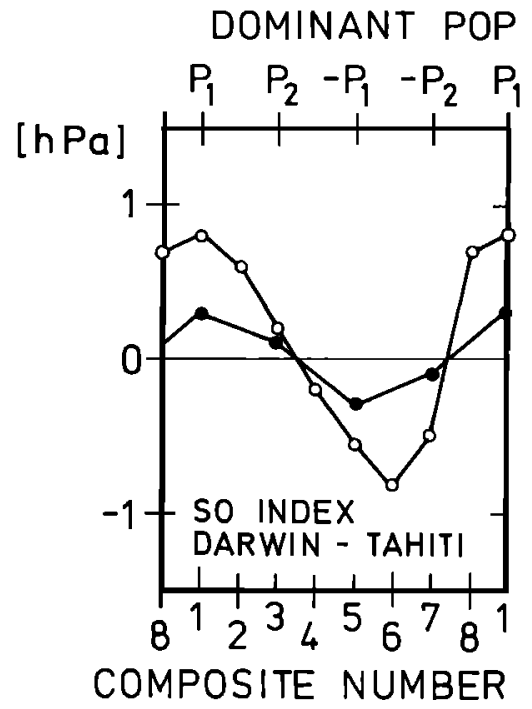

Fig. 14. SO index calculated from associated composite (open circles) and correlation (solid circles) patterns of sea level pressure. The SO index is defined as the sea level pressure difference Tahiti minus Darwin.

pattern with maximum variance in the Western PacificIndonesian region, strongly resembling the 30- to 60-day waves observed in the atmosphere. The wave occurs over a fairly wide range of periods in the spectrum. The mean POP period of 24 days is smaller than that found in the observations, but it is consistent with the results of other GCM simulations.

The results derived from the POP analysis are consistent with those obtained from a frequency-wave number variance analysis, but they contain more information. An advantage of the POP method is that the pattern structure and form of propagation need not be specified a priori. The analysis yields the dominant oscillation patterns with associated frequencies, damping rates, and propagation properties as a single entity. Using standard Fourier analysis techniques, a superposition of many phase-correlated components would be required to provide the same information.

To clarify the detailed physical characteristics of the GCM tropical 30- to 60-day wave first identified through a POP analysis of the equatorial $200-\mathrm{hPa}$ velocity potential, further fields were analyzed in terms of their associated correlation and composite patterns. The correlation and composite pattern techniques yield approximately the same information.
Composites have the advantage that they are able to identify phase asymmetries, intermittency, and other non-Gausian features. The associated correlation pattern technique, on the other hand, produces a more condensed representation in terms of only two diagrams and is more consistent with the original POP analysis based on second moments only.

The associate pattern analysis of the fields of 200- and 850$\mathrm{hPa}$ tropical velocity potential, $200-\mathrm{hPa}$ stream function, sea level pressure, $10-\mathrm{m}$ wind, and convective rainfall yielded the following results:

1. The GCM 30- to 60-day wave is not limited to the equator, but extends over the entire tropical region. At the $200-\mathrm{hPa}$ level, both the velocity potential and the stream function exhibit well-organized zonal wave number 1 patterns, which are almost perfectly $90^{\circ}$ out of phase. This property has been noted by Hendon [1986] for the velocity potential and stream function of an equatorially trapped Kelvin wave for a linearized equatorial $\beta$-plane shallow water model [cf. Matsuno, 1966]. The stream function pattern is also in accordance with Knutson and Weickmann's [1987] analysis of observed data. The disturbance travels with an eastward phase velocity of more than $20 \mathrm{~m} \mathrm{~s}^{-1}$ over most of the global tropics, but it slows down over areas with significant precipitation (Indonesia, South America, Africa). Increasing (decreasing) propagation speed is accompanied by decreasing (increasing) wave amplitudes.

2. At the surface a significant signal is found in the sea level pressure and the zonal component of $10-\mathrm{m}$ wind. No regular space-time structure is found in the meridional $10-\mathrm{m}$ wind component. This may be due to the lack of seasonal stratification. The spatial pattern of the signal is consistent with Gill's [1980] study of the response of the tropical atmosphere to stationary equatorial forcing using a linear, equatorial $\beta$-plane model.

3. There is some indication of a relation between the amplitude, speed of propagation, and phase of the associated patterns for the $200-\mathrm{hPa}$ velocity potential and rainfall. During the stage in which the wave propagates slowly, its amplitude is high, and the rainfall and velocity potential are in phase, with a maximum over Indonesia. After a quarter of a period, the rainfall and velocity potential are no longer in phase, the spatial disturbance propagates more rapidly, and the amplitude has decreased.

It would be interesting to clarify theoretically why the propagation velocity and wave amplitude vary simultaneously and whether a causal relationship exists between the amplification and deceleration of the GCM 30- to 60-day wave on the one hand and the phase correlation of the upper air velocity po-

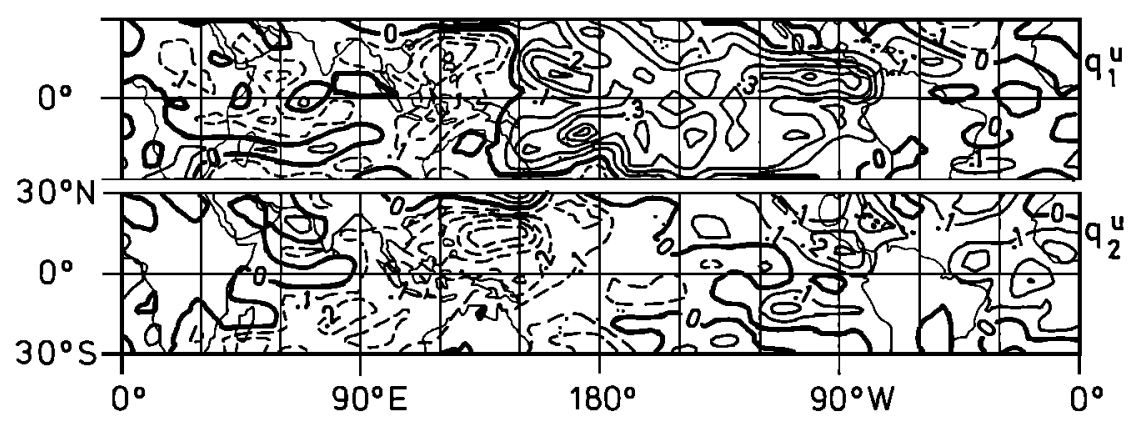

Fig. 15. Associated patterns $q_{1}{ }^{\prime \prime}$ and $q_{2}{ }^{\prime \prime}$ for surface zonal wind. Normalization as in Figure 11, with units of meters per second. 


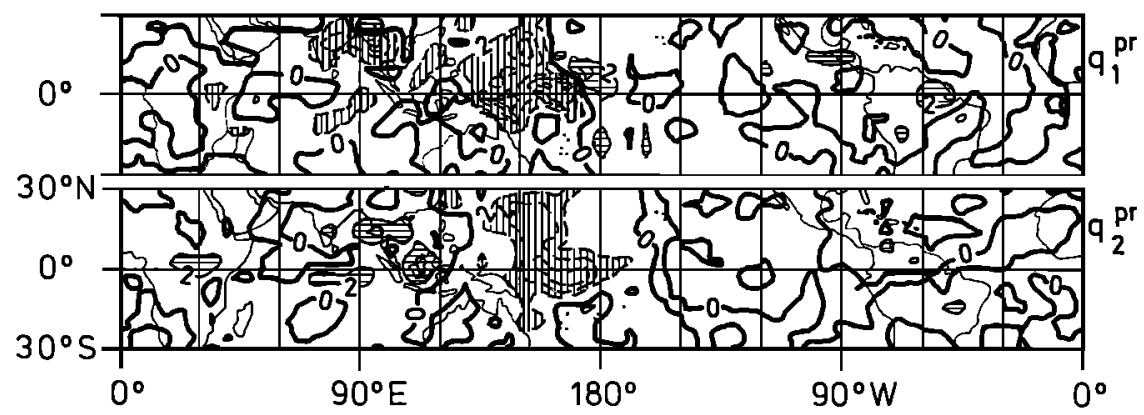

Fig. 16. Associated patterns $q_{1}{ }^{p r}$ and $q_{2}{ }^{p r}$ for convective rainfall. Areas with rainfall exceeding $2 \mathrm{~mm} \mathrm{day}^{-1}$ are hatched. Normalization as in Figure 11, with units of millimeters per day.

tential and rainfall patterns on the other hand, and, conversely, between the acceleration and damping of the wave and the lack of phase correlation between the velocity potential and rainfall.

As a possible explanation, we suggest that the equatorial 30- to 60-day wave is essentially a Kelvin wave, which can occur either as a forced wave or as a freely propagating wave. The forced time interval corresponds to periods when the minimum of the velocity potential lies in areas of maximum precipitation and the $p_{1}$ pattern is dominant. The forcing slows down the eastward migration, is associated with a high wave amplitude, and is accompanied by a longitudinally stationary rainfall anomaly. After the wave has propagated out of the area of maximum mean precipitation, the forcing and wave extrema are no longer in phase, and the wave is transformed to an almost free wave. The wave decays, and its phase speed increases. This stage is characterized by the pattern $p_{2}$.

\section{REFERENCES}

Barnett, T. P., Interaction of the monsoon and Pacific trade wind systems at inter-annual time scales, $\mathbf{I}$, The equatorial zone, Mon. Weather Rev., 111, 756-773, 1983.

Barnett, T. P., and R. W. Preisendorfer, Origins and levels of monthly and seasonal forecast skill for United States surface air temperatures determined by canonical correlation analysis, Mon. Weather Rev., 115, 1825-1850, 1987.

Bruns, T. and $H$. von Storch, Performance of the ECMWF-T21GCM in the tropics, Research Activities in Atmospheric and Oceanic modelling, edited by G. J. Boer, CAS/JSC Work. Group on Numer. Exp. Rep. 9, pp. 7.16-7.20, World Meteorol. Organ., Geneva, Switzerland, 1986

Chang, C. P., Viscous internal gravity waves and low frequency oscillations in the tropics, J. Atmos. Sci., 34, 901-910, 1977.

Chiba, M., 50-day oscillation of the tropical atmosphere simulated by a low resolution spectral AGCM, Research Activities in Atmospheric and Oceanic modelling, edited by G. J. Boer, CAS/JSC Work. Group on Numer. Exp. Rep. 9, pp. 7.4-7.5, World Meteorol. Organ., Geneva, Switzerland, 1986.

Fischer, G. (Ed), Climate simulations with the ECMWF T21 model in Hamburg, Large-Scale Atmos. Modelling Rep. I, 159 pp., Meteorol. Inst. der Univ. Hamburg, Hamburg, Federal Republic of Germany, 1987.

Geisler, J. E., and E. J. Pitcher, The 30-60 day oscillation in a perpetual January simulation with the NCAR general circulation model, Workshop on Comparison of Simulations by Numerical Models of the Sensitivity of the Atmospheric circulation to SST Anomalies, World Clim. Prog., Rep. 138, pp. 69-71, World Meteorol. Organ., Geneva, Switzerland, 1986.

Gill, A., Some simple solutions for heat-induced tropical circulation J. R. Meteorol. Soc., I06, 447-462,m 1980

Hasselmann, K., PIPs and POPs: The reduction of complex dynam- ical systems using principal interaction and oscillation patterns, $J$. Geophys. Res., this issue.

Hayashi, Y., Space time spectral analysis and its applications to atmospheric waves, J. Meteorol. Soc. Jpn., 60, 156-171, 1982.

Hayashi, Y., and D. G. Golder, Tropical intraseasonal oscillations appearing in a GFDL General Circulation Model and FGGE data, 1, Phase propagation, J. Atmos. Sci., 43, 3058-3067, 1986.

Hendon, H. H., Streamfunction and velocity potential representation of equatorially trapped waves, J. Atmos. Sci., 43, 3038-3042, 1986.

Horel, I D Complex nrincipal compnnent analysis Thenry and examples, J. Clim. Appl. Meteorol., 23, 1660-1673, 1984.

Knutson, T. R., and K. M. Weickmann, 30-60 day atmospheric oscillations: Composite life cycles of convection and circulation anomalies. Mon. Weather Rev., 115, 1407-1436, 1987

Knutson, T. R., K. M. Weickmann, and J. E. Kutzbach, Global-scale intraseasonal oscillations of outgoing longwave radiation and 250 $\mathrm{mb}$ zonalwind during northern hemisphere summer, Mon. Weather Rev., 114, 605-623, 1986.

Lau, K.-M, and P. H. Chan, Aspects of the 40-50 day oscillation during the northern winter as inferred from outgoing longwave radiation, Mon. Wearher Rev., 113, 1889-1909, 1987.

Lau, K.-M, and L. Peng, A preliminary theory of the origin of the "40-50" day oscillation, Workshop on Comparison of Simulations by Numerical Models of the Sensitivity of the Atmospheric Circulation to SST Anomalies, World Clim. Prog., Rep. 138, pp. 72-76, World Meteorol. Organ., Geneva, Switzerland, 1986.

Lau, K.-M, and T. Phillips, Coherent fluctuations of extratropoical geopotential height and tropical convection on intraseasonal time scales, J. Atmos. Sci., 43, 1164-1181, 1986.

Lau, N. C., Diagnosis of intra-seasonal oscillations appearing in GCM experiments conducted at GFDL. Workshop on Comparison of Simulations by Numerical Models of the sensitivity of the atmospheric circulation to SST anomalies, World Clim. Prog. Rep. 138, pp. 63-68, World Meteorol. Organ., Geneva, Switzerland 1986.

Lau, N. C., and K. M. Lau, The structure and propagation of intraseasonal oscillations appearing in a GFDL general circulation model, J. Atmos. Sci., 43, 2023-2047, 1986.

Lorenc, A. C., The evolution of planetary scale $200 \mathrm{mb}$ divergences during the FGGE year, Q. J. R. Meteorol. Soc., 110, 427-441, 1984.

Luksch, U., H. von Storch, and Y. Hayashi, Monte Carlo experiments with frequency-wavenumber spectra, Rep. 10, Max-Planck-Institut für Meteorol., Hamburg, Federal Republic of Germany, 1987.

Madden, R. A., Seasonal variations of the $40-50$ day oscillation in the tropics, J. Atmos. Scl., 43, 3138-3158, 1986.

Madden, R. A., and P. R. Julian, Detection of a 40-50 day oscillation in the zonal wind in the tropical Pacific, J. Atmos. Sci., 28, 702-708, 1971.

Madden, R. A., and P. R. Julian, Description of global-scale circulation cells in the tropics with a 40-50 day period, J. Atmos. Sci. $29,1109-1123,1972$.

Matsuno, T., Quasi-geostrophic motions in the equatorial area, $J$. Meteorol. Soc. Jpn., 44, 25-42, 1966.

Preisendorfer, R. W., and T. P. Barnett, Significance tests for empirical orthogonal functions, paper presented at Fifth Conference on Probability and Statistics, Am. Meteorol. Soc., Las Vegas, Nev., Nov. 15-18, 1977. 
Preisendorfer, R. W., F. W. Zwiers, and T. P. Barnett, Foundations of Principal Component Selection Rules, SIO Ref. Ser. pp. 81-84, Scripps Inst. of Oceanogr., Univ. of Calif., San Diego, 1981.

Swinbank, R., Simulation of intraseasonal oscillation in GCM experiments, Research Activities in Atmospheric and Oceanic Modelling, edited by G. J. Boer, CAS/JSC Work. Group on Numer. Exp. Rep. 9, pp. 7.1-7.3, World Meteorol. Organ., Geneva, Switzerland, 1986. von Storch, H., Numerische Filter zur Dämpfung meteorologischen Lärms in hemisphärischen Modellen, Hamb. Geophys. Einzelschriften 44, Meteorol. Inst. der Univ. Hamburg, Hamburg, Federal Republic of Germany, 1980

Weickmann, K. M., G. L. Lussky, and J. E. Kutzbach, Intraseasonal
(30-60 day) fluctuations of outgoing longwave radiation and 250 mb streamfunction during northern winter, Mon. Weather Rev., $113,941-961,1985$.

T. Bruns, I. Fischer-Bruns, K. Hasselmann, and H. von Storch, Max-Plank-Institut für Meteorologie, Bundstrasse 55, D-2000 Hamburg 13, Federal Republic of Germany.

(Received September 18, 1987; revised April 12, 1988; accepted April 13, 1988.) 